\title{
Effect of distinct environmental actions on the durability of RC slabs strengthened with prestressed CFRP laminate strips
}

\author{
J. Sena-Cruz, L. Correia, G. Escusa, E. Pereira \\ ISISE, University of Minho, Guimarães, Portugal
}

J. Michels

Empa, Dübendorf, Switzerland

P. M. França

CCCEE, University of Madeira, Funchal, Portugal

\begin{abstract}
In the context of concrete structures strengthening, the externally bonded reinforcement (EBR) technique is the most widely used strategy. By prestressing the FRP materials attached to the concrete substrate, the ad-vantages of external prestressing and of the EBR technique are combined. The present work investigates the durability of reinforced concrete (RC) slabs strengthened with prestressed CFRP strips according the EBR technique. Two different anchorage systems were studied: (i) the Mechanical Anchorage (MA) and (ii) Gradient Anchorage (GA). The durability of the specimens was evaluated by exposing them to one out of four environmental conditions, this combined with a sustained load of $1 / 3$ of the ultimate load for a period of 8 months. Finally, the slabs were monotonically tested up to failure by using a four-point bending test configuration. This work presents the general description of the experimental program, the analysis of the main results, as well as retained conclusions.
\end{abstract}

\section{INTRODUCTION}

The present work aims at contributing for a better understating the durability related aspects of $\mathrm{RC}$ slabs strengthened with prestressed EBR CFRP laminates. In this sense, an experimental program was carried out where the type of end anchorage system, the environmental condition and the loading scheme were the main variables studied. After being subjected to the specified environmental conditions and the prescribed loading scheme for a period of eight months, the slabs were monotonically tested up to failure, under displacement control and using the four-point bending test configuration. The influence of both the environmental conditions, and the cracking developed due to the sustained load on the overall performance of the prestressing systems are also discussed in the present work.

\section{EXPERIMENTAL PROGRAM}

The experimental program included nineteen $\mathrm{RC}$ slabs: (i) one reference specimen (REF_T0), (ii) nine specimens strengthened with the mechanical anchorage (MA) system and (iii) nine specimens strengthened using the gradient anchorage (GA) system. All specimens were labelled with a generic denomination $X_{-} Y \_Z$, where $X$ represents the specimen's type of anchorage (MA or GA), $\mathrm{Y}$ the environmental condition (REF for laboratory premises, $\mathrm{W}$ for the immersion in water, $\mathrm{CW}$ for the immersion in water with chlorides and WD for the wet-dry cycles) and Z the cracking state ( $\mathrm{U}$ for uncracked and $\mathrm{C}$ for cracked). It should be mentioned that three slabs (REF_T0, MA_REF_T0 and GA_REF_T0) were tested before the application of the environmental action/loading. For the remaining ones, one half was subjected the different environmental actions (labelled by _U) and the other to the combined effect of environmental and loading actions (labelled by_C).

Figure 1 shows the specimens' geometry and the test configuration used in the experimental program.

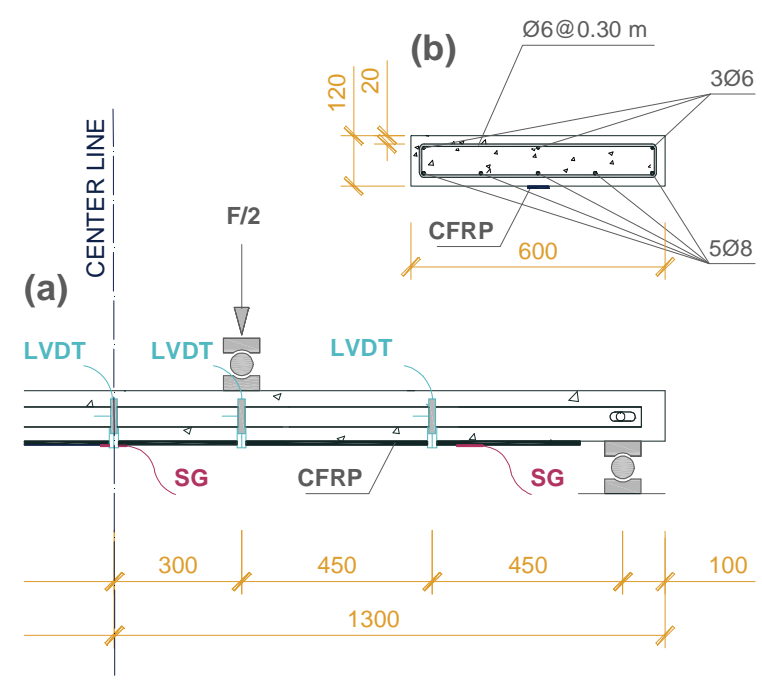

Figure 1. (a) Geometry and test configuration; (b) cross-section. Note: all units in [mm]. 


\section{RESULTS}

The test of the specimen REF was stopped when the midspan deflection was $100 \mathrm{~mm}$ due to the LVDT measurement range limitation. The failure mode would most likely be in this case concrete crushing on the top fiber at midspan. The specimen MA_REF failed by CFRP rupture in tension when their strain at the midspan attained at about $1.5 \%$. The failure mode of the remaining slabs was the intermediate debonding, cohesive at the concrete. Flexural cracks contributed to the debonding, which progressively shifted towards the strip ends.

Digital image correlation (DIC) endorsed this analysis. Figure 2 shows the crack evolution on the bottom surface of a typical MA slab, in terms of maximum principal strains, whereas Figure 3 typical total load versus mid-span displacement.
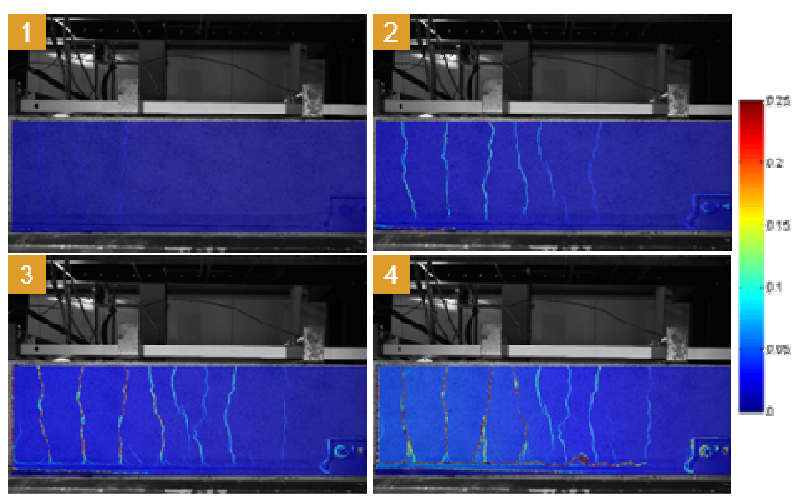

Figure 2. Principal tensile strain fields mapped during testing using digital image correlation on MA_W_U slab.

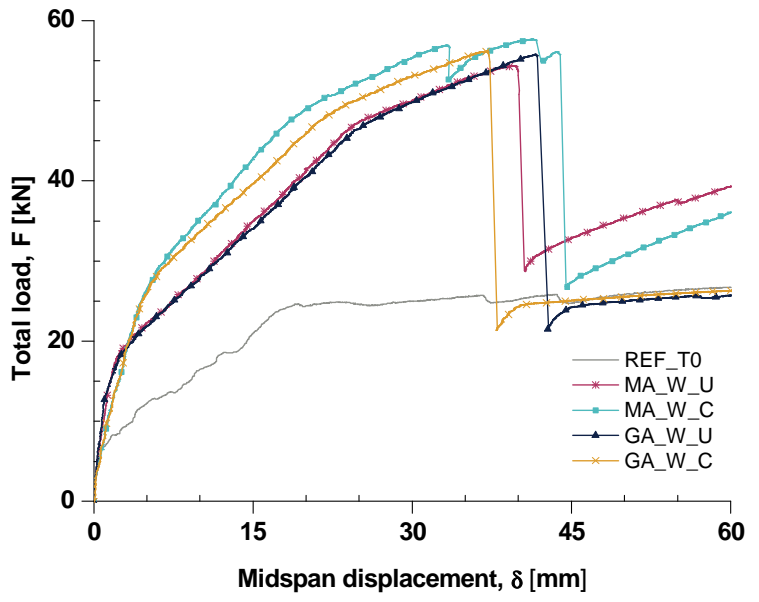

Figure 3. Typical total load versus mid-span displacement (series of specimens immersed in water).

Figure 4 resumes the key results of the experimental program. As expected, all four environmental actions had a negative effect on the yielding $\left(\delta_{y}, F_{y}\right)$ and on the ultimate $\left(\delta_{\mathrm{u}}, \mathrm{F}_{\mathrm{u}}\right)$ levels of both GA and MA specimens. In average terms, the strengthened "T0" specimens had considerable higher cracking, yielding and ultimate loads. Also, specimens submitted to the 8 months aging showed an average yielding and failure for lower deflection levels.

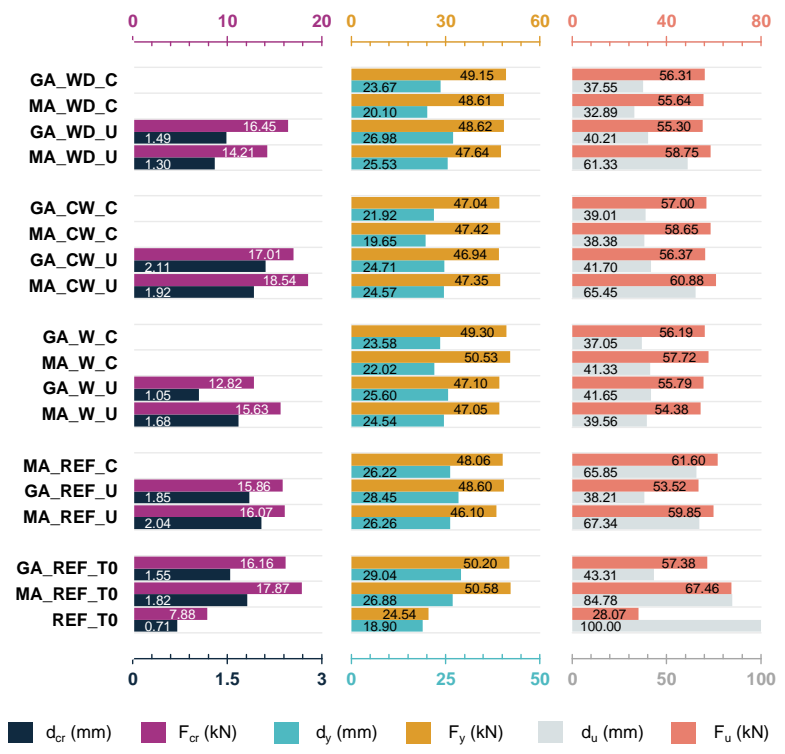

Figure 4. Key results obtained from the experimental program.

\section{CONCLUSIONS}

The performed tests led to the following main conclusions:

- A similar response was observed for both anchorage techniques, yet the mechanical anchors of the MA system prevented a premature failure and allowed the slabs to support greater ultimate loads and deflections;

- The MA_REF_T0 slab was the only that failed by FRP rupture at its maximum tensile capacity, whereas the remaining strengthened slabs seemed to fail by intermediate debonding (cohesive in the concrete);

- The evolution of the crack pattern described using the Digital Image Correlation methodology helped to understand that the debonding started at midspan of the slab and then moved to the CFRP laminate extremities;

- All environmental actions caused a reduction of the yielding and failure loads of both anchorage systems, full immersion in water and sea-water being the most severe ones. However, the influence of each environment was different on each anchorage system;

The synergetic actions (cracking + environment) caused the highest degradation of the load carrying capacity of the slabs.

\section{ACKNOWLEDGEMENTS}

This work is supported by FEDER funds through the Operational Program for Competitiveness Factors COMPETE and National Funds through FCT - Portuguese Foundation for Science and Technology under the project FRPreDur - FCOMP-01-0124FEDER-028865. The second author also wishes to acknowledge the FCT grant SFRH/BD/99309/2013. 